\title{
Cystic fibrosis: The urgent need to report on mutations among patients of African descent
}

In this $S A R J$ issue, Mphahlele et al. ${ }^{[1]}$ have reported on a condition that is being increasingly diagnosed in people of African descent: cystic fibrosis (CF). The article will contribute to rectifying the misconception that this condition is restricted to people of European descent and underscore the need to further investigate the mutations specific to people of African descent. The delayed age of diagnosis, specifically among patients of African descent, further illustrates the need to educate health professionals, to improve their awareness and raise their index of suspicion of CF in patients of African ancestry. The research also emphasises the need to initiate newborn screening for $\mathrm{CF}$, which has been shown to be critical in identifying the condition in non-white patients in the USA. ${ }^{[2]}$

In the Mphahlele et al.$^{[1]}$ study, the absence of any detected mutation in almost all the non-white patients is consistent with the literature, as this is expected with the currently used 30-mutation CFTR panel for which the diagnosis rate of $\mathrm{CF}$ in non-European populations is very low. ${ }^{[2]}$ However, mutations in CFTR in South Africans of black African ancestry were first reported 2 decades ago; ${ }^{[3]}$ a specific mutation found in black South Africans, $3120+1 G>A$, which was initially reported in three African-American CF patients, has been shown to account for $9-14 \%$ of African-American CF chromosomes. ${ }^{[4]}$ This mutation $(3120+1 G>A)$ is now routinely tested for in selected medical genetic settings in South Africa (SA). ${ }^{[5]}$ The investigation of specific CFTR mutations in various populations of non-European ancestry is gaining increasing attention globally, especially in countries that, like SA, have a population of diverse ethnic background, such as Reunion Island, ${ }^{[6]} \mathrm{Brazil}^{[7]}$ and the USA. ${ }^{[2]}$ This research is shedding light on ethnic-specific variants that cannot be detected by the routinely used 30-mutation CFTR panel. It is, therefore, reasonable to call for urgent further studies using the full sequencing of the CFTR gene in patients of non-European ancestry in SA, in Africa and globally, to reveal more specific mutations that will increase the diagnostic validity and yield of the molecular testing of the CFTR gene.

A well-designed CFTR expanded panel using ethnic-specific variants is desirable to improve $\mathrm{CF}$ carrier detection rates within specific populations in SA, specifically in populations of African ancestry.

\section{Ambroise Wonkam}

Division of Human Genetics, Department of Medicine, Faculty of Health Sciences, University of Cape Town, South Africa

ambroise.wonkam@uct.ac.za

1. Mphahlele REM, Naidoo V, Thula S, Masekela R. An analysis of outcomes in children with cystic fibrosis in a tertiary African centre: A retrospective study. S Afr Respir J 2016;22(2):35-37. DOI:10.7196/SARJ.2016.v22i2.76

2. Pique L, Graham S, Pearl M, Kharrazi M, Schrijver I. Cystic fibrosis newborn screening programs: Implications of the CFTR variant spectrum in non-white patients. Genet Med 2016;May. DOI:10.1038/gim.2016.48

3. Carles S, Desgeorges M, Goldman A, et al. First report of CFTR mutations in black cystic fibrosis patients of southern African origin. J Med Genet 1996;33(9):802-804. DOI:10.1136/jmg.33.9.802

4. Padoa C, Goldman A, Jenkins T, Ramsay M. Cystic fibrosis carrier frequencies in populations of African origin. J Med Genet 1999;36(1):41-44. DOI:10.1111/j.1399-0004.1994.tb04405.x

5. Goldman A, Graf C, Ramsay M, Leisegang F, Westwood AT. Molecular diagnosis of cystic fibrosis in South African populations. S Afr Med J 2003;93(7):518-519.

6. Marion H, Natacha G, Brigitte M, et al. The p.Gly622Asp (G622D) mutation, frequently found in Reunion Island and in black populations, is associated with a wide spectrum of CF and CFTR-RD phenotypes. J Cyst Fibros 2015;14(3):305-309.

7. Faucz FR, Souza DA, Olandoski M, Raskin S. CFTR allelic heterogeneity in Brazil: Historical and geographical perspectives and implications for screening and counselling for cystic fibrosis in this country. J Human Genet 2010;55(2):71-76

S Afr Respir J 2016;22(2):34. DOI:10.7196/SARJ.2016.v22i2.78 